\title{
The Value of Postpartum Pelvic Floor Rehabilitation Nursing to Prevent Pelvic Floor Dysfunction
}

\author{
Cheng Li, Qiu Baohua, Huang Xinke* \\ Department of gynaecology and obstetrics, the First Affiliated Hospital of Jinan University, Guangzhou, China \\ Email address: \\ 929901838@qq.com (Huang Xinke) \\ ${ }^{*}$ Corresponding author \\ To cite this article: \\ Cheng Li, Qiu Baohua, Huang Xinke. The Value of Postpartum Pelvic Floor Rehabilitation Nursing to Prevent Pelvic Floor Dysfunction. \\ Journal of Gynecology and Obstetrics. Vol. 9, No. 2, 2021, pp. 50-53. doi: 10.11648/j.jgo.20210902.15
}

Received: March 9, 2021; Accepted: March 22, 2021; Published: March 30, 2021

\begin{abstract}
Female pelvic floor dysfunctions are the most common diseases after pregnancy. Vaginal relaxation, stress urinary incontinence (SUI), and pelvic organ prolapse (POP), decreased sexual activity, and mental depression and other clinical symptoms, are the most normal results of childbirth which leading to cause various problems for the mother. We aim to explore the clinical situation and effect of postpartum pelvic floor rehabilitation nursing for patients with postpartum pelvic floor dysfunction. In this study, 100 patients with postpartum pelvic floor dysfunction randomly selected from January 2018 to December 2019. They were randomly divided into two groups for nursing care, including control group and observation group. The control group received routine care, and the observation group received postpartum pelvic floor rehabilitation care. We carry out comparison of the clinical nursing effects of the two groups. After analysis and statistics, the total clinical effectiveness $(94.0 \%)$ and nursing satisfaction $(92.0 \%)$ of the observation group were significantly higher than those of the control group $(80.0 \%, 72.0 \%)$, and the differences between the groups were significant $(\mathrm{P}<0.05)$. In conclusion, implementing pelvic floor rehabilitation nursing for patients with postpartum pelvic floor dysfunction can better promote the rehabilitation of patients' pelvic floor function, improve clinical effects and nursing satisfaction.
\end{abstract}

Keywords: Pelvic Floor Dysfunction, Postpartum Pelvic Floor Rehabilitation Nursing, Clinical Effect

\section{Introduction}

Female pelvic floor dysfunction (FPFD) mainly refers to the patient's pelvic floor support failure or loss due to childbirth, which leads to vaginal relaxation, stress urinary incontinence (SUI), and pelvic organ prolapse (POP), decreased sexual activity, and mental depression and other clinical symptoms [1]. Pelvic floor dysfunction can cause great harm to patients $[2,3]$. If nursing care is not resumed in time, it is easy to cause other complications, which will seriously affect the physical health, psychological status and daily life of patients. The postpartum puerperium is a critical period for maternal rehabilitation [4]. Postpartum rehabilitation nursing can promote the improvement of pelvic floor function to a certain extent by guiding the exercise pelvic floor, and has the advantages of simplicity and ease of learning $[5,6]$. However, some parturients have aggravated the condition due to the wrong contraction of the abdominal muscles, which affects the rehabilitation effect $[6,7]$. The bioelectrical stimulation therapy instrument is a physical therapy instrument [8], which mainly promotes passive muscle contraction through electrical stimulation and synergistically improves the pelvic floor muscle function $[9,10]$. This study analyzed the clinical nursing effect of postpartum pelvic floor rehabilitation nursing on pelvic floor dysfunction.

\section{Materials and Methods}

\subsection{General Data}

The research data were randomly selected from 100 patients who had natural delivery in the gynecology department of our hospital and had postpartum pelvic floor dysfunction from January 2018 to December 2019. The ages of the patients were in the range of 23 to 33 years, with an average age of (27.4 \pm 2.6$)$ years. All were primiparous, gave birth at term, and all were singletons. The patients were randomly divided into two groups by the digital table method, namely the control 
group, with 50 cases, all in the range of 23 to 33 years old, with an average age of $(27.1 \pm 2.9)$ years. The 50 cases in the observation group were all in the range of 23 to 33 years old, with an average age of $(27.8 \pm 2.3)$ years. Both groups of patients were diagnosed with postpartum pelvic floor dysfunction, and no complications such as postpartum hemorrhage occurred. There is no significant difference in the comparison results in terms of general medical record data (age, mode of delivery, type of disease, and clinical manifestations) ( $\mathrm{P}>0.05)$, which is not statistically significant and can be compared in clinical research.

\subsection{Methods}

\subsubsection{Control Group}

Control group Give patients routine care. Specific: (1) Basic nursing: Medical staff should closely observe and record the clinical signs and symptoms of the patient, report abnormalities discovered in a timely manner to the relevant attending physician, and take active and effective methods for correct handling. (2) Psychological intervention: Medical staff should be patient, meticulous and considerate to communicate with them, understand the concerns of patients, and explain the prognostic effects and successful cases of pelvic floor treatment try to eliminate the patients' negative emotions such as tension, fear, and worry, and give them full spiritual encouragement, comfort and support to help patients establish a positive concept of overcoming the disease, so that patients can maintain a relaxed, optimistic, and positive attitude to actively cooperate with doctors for treatment. (3) Diet intervention: Medical staff should instruct patients to drink more water, eat more vegetables, fruits, and light, digestible and nutritious food to avoid problems such as constipation.

\subsubsection{Observation Group}

Patients in the observation group were given postpartum pelvic floor rehabilitation care, that is, to increase their pelvic floor rehabilitation training on basis of routine care. (1) The nurse conducts anal retraction check on the patient, and develops an individualized rehabilitation plan based on the results; (2) The nurse guides the patient to perform bioelectric stimulation rehabilitation training, that is, inserts a disposable treatment rod in the patient's vagina and connects it to electronic biofeedback treatment instrument. We select the appropriate amount of electrical stimulation to train the patient's pelvic floor muscles for myoelectric activity response training, and adjust the training intensity at any time according to the observation results; (3) The nursing staff guide the patient to take conscious pelvic floor muscle active retracting training before going to bed and rest. That is, two automatic contraction trainings of urethra, perineum and anus are carried out every day, each contraction time is $5 \mathrm{~s}-10 \mathrm{~s}$, the interval is $3 \mathrm{~s}-5 \mathrm{~s}$, the repetitive training time is $5 \mathrm{~min}$ each time, and the continuous training is more than 8-10 weeks.

\subsection{Statistical Analysis}

The clinical nursing effect and satisfaction of 100 patients with pelvic floor dysfunction were statistically compared through the software SPSS20.0. Counting data adopts $\mathrm{X}^{2}$ inspection. When $\mathrm{P}<0.05$, the comparison is statistically significant.

\section{Results}

\subsection{Compare the Nursing Effect of the Two Groups}

As shown in Table 1, after nursing, the total effective rate of clinical care for patients in the control group is $80.00 \%$, and the total effective rate of clinical care for patients in the observation group is $94.00 \%$. The observation group is significantly higher than the control group, $\mathrm{P}<0.05$. The difference between the two groups was statistically significant.

Table 1. Comparison of clinical nursing effects between the two groups of patients.

\begin{tabular}{|c|c|c|c|c|}
\hline Group & control group & observation group & $\mathrm{X}^{2}$ value & P value \\
\hline Case (n) & 50 & 50 & - & - \\
\hline Cured (n, \%) & $20(40.00)$ & $28(56.00)$ & 1.847368 & $>0.05$ \\
\hline Significantly effective (n, \%) & $6(12.00)$ & $11(22.00)$ & 2.307692 & $>0.05$ \\
\hline Effective (n, \%) & $14(28.00)$ & $8(16.00)$ & 1.880424 & $>0.05$ \\
\hline Invalid (n, \%) & $10(20.00)$ & $3(6.00)$ & 4.129412 & $<0.05$ \\
\hline Total effective rate $(\mathrm{n}, \%)$ & $40(80.00)$ & $47(94.00)$ & 4.129412 & $<0.05$ \\
\hline
\end{tabular}

\subsection{Compare the Nursing Satisfaction of the Two Groups}

As shown in Table 2, after statistical analysis, the total satisfaction with clinical care of the control group and the observation group were $72.0 \%$ and $92.0 \%$, respectively. The difference between the two groups was significant $(\mathrm{P}<0.05)$, and there was a certain statistical significance.

Table 2. Comparison of nursing satisfaction between the two groups of patients.

\begin{tabular}{llll}
\hline Group & Control group & Observation group & $\mathbf{X}^{\mathbf{2}}$ value \\
\hline Case (n) & 50 & 50 & P value \\
Very satisfied (n, \%) & $23(46.00)$ & $35(70.00)$ & - \\
Satisfied (n, \%) & $13(26.00)$ & $11(22.00)$ & 4.254545 \\
Not satisfied (n, \%) & $14(28.00)$ & $4(8.00)$ & 0.069581 \\
Total satisfaction (n, \%) & $36(72.00)$ & $46(92.00)$ & 5.571429 \\
\hline
\end{tabular}




\section{Discussions}

Pelvic floor dysfunction is a complication that often occurs in patients after delivery $[11,12]$. It mainly refers to the relaxation of the pelvic floor muscles caused by the patient's breath-holding force and excessive pulling of the pelvic floor tissue during the natural delivery process [13]. It has a serious impact on the postpartum recovery of patients. Therefore, clinically, it is necessary to strengthen the nursing intervention for the recovery of postpartum pelvic floor function [14]. Pelvic floor rehabilitation care is a series of recovery training treatments for patients with postpartum pelvic floor muscle function decline [15]. It can effectively repair damaged pelvic floor muscles, and has no pain [16]. In addition, it was convenient and simple operation, high compliance and low cost [15].

This study showed that 50 randomly selected patients with postpartum pelvic floor dysfunction who received postpartum pelvic floor rehabilitation after clinical care had the total clinical care efficiency $(94.0 \%)$ and total nursing satisfaction $(92.0 \%)$. Patients in observation group were significantly better than those who used conventional care $(80.0 \%, 72.0 \%)$, and the difference was statistically significant $(\mathrm{P}<0.05)$.

Previous studies have compared the application effects of early rehabilitation nursing and late rehabilitation nursing in postpartum pelvic floor function rehabilitation [17]. The results of the study show that the advantages of early rehabilitation nursing are more prominent, reflected in the following aspects: First, the incidence of urinary incontinence and organs prolapse was lower; Second, sexual satisfaction was higher; Third, rehabilitation compliance and nursing satisfaction are higher; Fourth, negative emotions such as anxiety and depression were significantly improved, which fully reflects the clinical practice of early rehabilitation care Value. It can be seen from the results that the implementation of early rehabilitation care can effectively prevent and control the occurrence of urinary incontinence and organ prolapse, and improve the quality of life of patients. This is mainly because the frequency pelvic floor rehabilitation therapy instrument and pelvic floor muscle training can allow patients to correctly contract and contract related muscles, promote the recovery of pelvic floor muscle strength [18]. Thereby, it could improve the sensitivity of pelvic floor muscles and nerves, and improve the patient's quality of sexual life [19].

\section{Conclusion}

In summary, the rehabilitation of pelvic floor care for patients with postpartum pelvic floor dysfunction can effectively promote the recovery of their pelvic floor function, improve the effectiveness of clinical care, and improve the clinical symptoms of patients, so as to better help patients recover as soon as possible.

\section{References}

[1] Wallace SL, Miller LD, Mishra K: Pelvic floor physical therapy in the treatment of pelvic floor dysfunction in women. Curr Opin Obstet Gynecol 2019, 31 (6): 485-493.

[2] Larouche M, Brotto LA, Koenig NA, Lee T, Cundiff GW, Geoffrion R: Depression, Anxiety, and Pelvic Floor Symptoms Before and After Surgery for Pelvic Floor Dysfunction. Female Pelvic Med Reconstr Surg 2020, 26 (1): 67-72.

[3] Himmler M, Kohl M, Rakhimbayeva A, Witczak M, Yassouridis A, Liedl B: Symptoms of voiding dysfunction and other coexisting pelvic floor dysfunctions: the impact of transvaginal, mesh-augmented sacrospinous ligament fixation. Int Urogynecol J 2021.

[4] Romano M, Cacciatore A, Giordano R, La Rosa B: Postpartum period: three distinct but continuous phases. J Prenat Med 2010 4 (2): $22-25$.

[5] Shishido E, Shuo T, Takahata K, Horiuchi S: Changes in salivary oxytocin levels and bonding disorder in women from late pregnancy to early postpartum: A pilot study. PLoS One 2019, 14 (9): e0221821.

[6] Thabet AA, Alshehri MA: Efficacy of deep core stability exercise program in postpartum women with diastasis recti abdominis: a randomised controlled trial. J Musculoskelet Neuronal Interact 2019, 19 (1): 62-68.

[7] Gluppe SL, Hilde G, Tennfjord MK, Engh ME, Bo K: Effect of a Postpartum Training Program on the Prevalence of Diastasis Recti Abdominis in Postpartum Primiparous Women: A Randomized Controlled Trial. Phys Ther 2018, 98 (4): 260-268.

[8] Kai H, Yamauchi T, Ogawa Y, Tsubota A, Magome T, Miyake T, Yamasaki K, Nishizawa M: Accelerated Wound Healing on Skin by Electrical Stimulation with a Bioelectric Plaster. Adv Healthc Mater 2017, 6 (22).

[9] Hwang UJ, Kwon OY, Lee MS: Effects of surface electrical stimulation during sitting on pelvic floor muscle function and sexual function in women with stress urinary incontinence. Obstet Gynecol Sci 2020, 63 (3): 370-378.

[10] Jha S, Walters SJ, Bortolami O, Dixon S, Alshreef A: Impact of pelvic floor muscle training on sexual function of women with urinary incontinence and a comparison of electrical stimulation versus standard treatment (IPSU trial): a randomised controlled trial. Physiotherapy 2018, 104 (1): 91-97.

[11] Herbert J: Pregnancy and childbirth: the effects on pelvic floor muscles. Nurs Times 2009, 105 (7): 38-41.

[12] Colla C, Paiva LL, Ferla L, Trento MJB, de Vargas IMP, Dos Santos BA, Ferreira CF, Ramos JGL: Pelvic floor dysfunction in the immediate puerperium, and 1 and 3 months after vaginal or cesarean delivery. Int J Gynaecol Obstet 2018, 143 (1): 94-100.

[13] Urbankova I, Grohregin K, Hanacek J, Krcmar M, Feyereisl J, Deprest J, Krofta L: The effect of the first vaginal birth on pelvic floor anatomy and dysfunction. Int Urogynecol J 2019, 30 (10): 1689-1696. 
[14] Hay-Smith J, Morkved S, Fairbrother KA, Herbison GP: Pelvic floor muscle training for prevention and treatment of urinary and faecal incontinence in antenatal and postnatal women. Cochrane Db Syst Rev 2008 (4).

[15] Lawson S, Sacks A: Pelvic Floor Physical Therapy and Women's Health Promotion. J Midwifery Womens Health 2018, 63 (4): 410-417.

[16] Ghaderi F, Bastani P, Hajebrahimi S, Jafarabadi MA, Berghmans B: Pelvic floor rehabilitation in the treatment of women with dyspareunia: a randomized controlled clinical trial. Int Urogynecol J 2019, 30 (11): 1849-1855.

[17] Deffieux X, Vieillefosse S, Billecocq S, Battut A, Nizard J,
Coulm B, Thubert T: Postpartum pelvic floor muscle training and abdominal rehabilitation: Guidelines. J Gynecol Obstet Biol Reprod (Paris) 2015, 44 (10): 1141-1146.

[18] Eyjolfsdottir H, Ragnarsdottir M, Geirsson G: Pelvic floor muscle training with and without functional electrical stimulation as treatment for stress urinary incontinence. Laeknabladid 2009, 95 (9): 575-581.

[19] Yang S, Sang W, Feng J, Zhao H, Li X, Li P, Fan H, Tang Z, Gao L: The effect of rehabilitation exercises combined with direct vagina low voltage low frequency electric stimulation on pelvic nerve electrophysiology and tissue function in primiparous women: A randomised controlled trial. J Clin Nurs 2017, 26 (23-24): 4537-4547. 